\section{Journal of \\ Epilepsy and \\ Clinical \\ Neurophysiology}

J Epilepsy Clin Neurophysiol 2010;16(4):143-148

\title{
Avaliação Neurofisiológica das Vias Auditivas e do Equilíbrio na Afasia de Broca - Apresentação de um caso ilustrativo
}

\author{
Bianca Simone Zeigelboim ${ }^{\mathrm{a}}$, Karlin Fabianne Klagenberga, Paulo Breno Noronha Liberalesso ${ }^{\mathrm{a}, \mathrm{b}, \mathrm{c}}$, \\ Priscila Menezes $^{\mathrm{a}}$, Daniele Vaz Gonçalves ${ }^{\mathrm{a}}$ \\ Universidade Tuiuti do Paraná, PR, Brasil
}

\begin{abstract}
RESUMO
Introdução: Afasia é uma alteração neurológica decorrente de uma lesão em áreas cerebrais que são responsáveis pela linguagem. Frequentemente, a afasia ocorre como resultado de um acidente vascular cerebral, comprometendo a linguagem de expressão (afasia de Broca), a linguagem de entendimento (afasia de Wernicke) ou ambas (afasia mista). A avaliação otoneurológica corresponde a um grupo de procedimentos para avaliação sequencial do sistema auditivo e vestibular a de suas relações com o sistema nervoso central. O sistema auditivo e vestibular são de grande importância no controle dos mecanismos auditivos centrais, no processamento auditivo central, no controle do senso de movimento e no equilíbrio. Objetivo: Nosso objetivo foi apresentar a sequência da avaliação neurológica das vias auditivas (periféricas e centrais) e do sistema vestibular em um paciente com afasia de Broca. Métodos: $\mathrm{O}$ paciente foi submetido a avaliação otorrinolaringológica, avaliação auditiva (audiometria tonal limiar convencional), testes de imitância acústica, pesquisa do reflexo acústico, avaliação do processamento auditivo central e do sistema vestibular. Conclusão: Nossos dados demonstram a importância da avaliação neurofisiológica das vias auditivas e do sistema vestibular em pacientes com afasia de Broca, para aprimorar o diagnóstico, tratamento e o acompanhamento evolutivo.
\end{abstract}

Unitermos: Afasia de Broca, vias auditivas, sistema vestibular.

\begin{abstract}
Neurophysiologic evaluation of auditory pathways and the balance in Broca's aphasia - Presentation of illustrative case

Introduction: Aphasia is a neurological disorder that results from damage to areas of the brain that are responsible for language. Often, the aphasia occurs as the result of a stroke, that leads to language expression impairment (Broca's aphasia), language comprehension impairment (Wernicke's aphasia) or the association of both (mixed aphasia). The otoneurologic evaluation is a group of semiologic standardized procedures that evaluates the relationship between the auditory and vestibular system and the central nervous system. The auditory pathways and the vestibular system are very important for the control of the central auditory mechanisms, the central auditory processing and the sense of movement and balance controls. Objective: Our aim was to present the neurophysiologic evaluation of the auditory pathways (peripheral and central) and the vestibular system in patients with Broca's aphasia. Methods: The patient was submitted to otorrinolaringologic evaluation, audiologic evaluation (tonal audiometria conventional), acoustics immittance measurements, research of the acoustic reflex and central auditory processing as well as vestibular system evaluation. Conclusions: Our data demonstrate the importance of the auditory pathways and the vestibular system neurophysiologic evaluation in patients with Broca's aphasia in order to improve the diagnosis, treatment and the follow-up.
\end{abstract}

Keywords: Broca's aphasia, auditory pathways, vestibular system.

a Pós-graduação em Distúrbios da Comunicação da Universidade Tuiuti do Paraná, PR, Brasil.

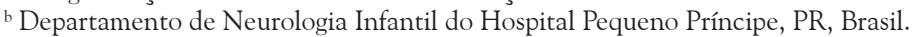

c Laboratório de EEG Digital do Hospital da Cruz Vermelha Brasileira, PR, Brasil.

Received Mar. 30, 2010; accepted July 16, 2010. 


\section{INTRODUÇÃO}

Afasia é um distúrbio da linguagem no qual há alteração de mecanismos linguísticos produtivos (relacionados à produção de fala) e interpretativos (relacionado à compreensão da fala), decorrendo, geralmente, de uma lesão cerebral estrutural como no caso dos acidentes vasculares cerebrais, traumatismos crânio-encefálicos, tumores cerebrais, entre outras. ${ }^{1}$ Quando a afasia é relacionada a uma lesão do córtex cerebral, pode ser acompanhada por alterações de outros processos cognitivos e sinais neurológicos, como hemiplegia, apraxia, agnosia, anosognosia e disfagia., ${ }^{2,3}$

As classificações das afasias são variadas e dependem da concepção de linguagem de cada autor. Os termos afasia anterior e posterior são utilizados denotando a localização da lesão em relação à fissura Sylviana. Os termos afasia de Broca, emissiva, de expressão e motora ${ }^{1}$ estão relacionados às principais características das afasias anteriores por envolverem de forma marcante os mecanismos de expressão e produção da linguagem verbal. Por outro lado, os termos afasia de Wernicke, receptiva, sensorial ${ }^{4}$ ou de compreensão, ${ }^{5}$ estão relacionados às lesões posteriores, sobretudo temporais e parietais, com marcado comprometimento na percepção da linguagem falada. A afasia mista ou global é caracterizada por alterações tanto na produção como na compreensão da linguagem falada. ${ }^{4}$

A neurofisiologia da audição pode ser dividida em três etapas: (a) atividade auditiva periférica; (b) atividade auditiva central e (c) processamento auditivo central (PAC). ${ }^{6}$

A avaliação do PAC deve ser sempre precedida pela avaliação da atividade auditiva periférica e da atividade auditiva central, que será realizada através de audiometria tonal limiar (estudo da capacidade de detecção de sons), timpanometria (estudo das condições de mobilidade do sistema tímpano-ossicular e verificação de presença ou ausência de reflexo acústico) e pesquisa dos percentuais de reconhecimento de fala (avaliação fisiológica da cóclea e do nervo auditivo). A identificação de alterações no PAC é de grande importância em pacientes com afasia, pois possibilita mudanças no processo de reabilitação fonoaudiológica. ${ }^{7}$

A alteração na expressão da linguagem falada nos pacientes com afasia pode alterar o resultado da avaliação do PAC, sendo indicado nestes casos, complementar a avaliação através da realização de um exame de potencial evocado auditivo de troco encefálico. ${ }^{8}$

A avaliação otoneurológica é um conjunto de procedimentos que permite a exploração semiológica dos sistemas auditivo e vestibular e de suas relações com o sistema nervoso central (SNC)..$^{9}$ A vestibulometria é um método de avaliação não invasivo cujos objetivos são: (a) auxiliar na confirmação do comprometimento vesti- bular; (b) reconhecer o lado lesado; (c) localizar a lesão (periférica, central ou mista); (d) verificar o tipo da lesão vestibular (irritativa ou deficitária); (e) caracterizar a intensidade da lesão (leve, moderada ou severa) e (f) monitorar a evolução do paciente vertiginoso. ${ }^{9}$

O objetivo de nosso estudo foi demonstrar a avaliação neurofisiológica das vias auditivas (periférica e central) e do equilíbrio em um paciente com afasia de Broca, ressaltando sua importância no diagnóstico, tratamento e acompanhamento evolutivo destes pacientes. A pesquisa foi realizada após autorização do paciente por meio de assinatura do Termo de Consentimento Livre e Esclarecido e após aprovação do Comitê de Ética e Pesquisa em Seres Humanos da Universidade Tuiuti do Paraná (parecer número: 008/2005).

\section{METODOLOGIA DA AVALIAÇÃO AUDITIVA E VESTIBULAR}

Breve relato do caso: Paciente do sexo masculino, 69 anos, branco, aposentado, casado e pai de três filhos. Possui grau de escolaridade de ensino fundamental. Em 2002 apresentou um acidente vascular cerebral (AVC) isquêmico decorrente de obstrução por trombo da artéria cerebral média esquerda. Evoluiu com afasia de Broca, caracterizada clinicamente por déficit na emissão da palavra falada, alterações fonético-fonológica; parafasias, alteração na organização sintática, na leitura e na escrita. Não apresenta alteração de compreensão. Após o AVC houve o surgimento de queixas de desequilíbrio. Apresenta como doenças de base hipertensão arterial sistêmica e hipercolesterolemia. $\mathrm{O}$ paciente refere zumbido na orelha esquerda desde os 18 anos de idade

Descrição sequencial dos métodos de avaliação: inicialmente foi realizada avaliação otorrinolaringológica com objetivo de excluir qualquer alteração que pudesse interferir na avaliação das vias auditivas. A avaliação audiológica foi realizada através de audiometria tonal limiar convencional com audiômetro de 2 canais (Madsen-GN Otometrics - modelo Itera ${ }^{\circledR}$ ), com fones TDH-39, com limiares em dB NA. O equipamento encontra-se calibrado de acordo com o padrão ISO 8253. A seguir, pesquisou-se a determinação do limiar de reconhecimento de fala (LRF) e do índice percentual de reconhecimento de fala (IPRF), em cabine acusticamente tratada. $\mathrm{O}$ grau e o tipo de perda auditiva foram caracterizados segundo as classificações de Davis e Silverman ${ }^{10}$ e Silman e Silverman. ${ }^{11}$

Foram realizadas medidas de imitância acústica para avaliar a integridade do sistema tímpano-ossicular por meio da curva timpanométrica e da pesquisa do reflexo acústico. O equipamento utilizado foi o impedanciômetro Madesn Otoflex $100^{\circledR}$. Para interpretação dos resultados, aplicaramse os critérios de Jerger. ${ }^{12}$ 
A avaliação do processamento auditivo central constituiu das seguintes etapas: (a) localização da fonte sonora; (b) memória sequencial para sílabas; (c) teste de escuta dicótica de dissílabos alternados (Staggered Spondaic Word Test - SSW); (d) teste de escuta monótica - PSI (Pediatric Speech Inteligibility - versão português); e (e) teste de detecção de intervalos aleatórios de silêncio (Random Gap Detection Test - RGDT). O SSW, PSI e o RGDT foram realizados com o auxílio de um discman, modelo D88, marca Sony ${ }^{\circledR}$, acoplado ao audiômetro. Foi utilizado o compact disc da obra Processamento Auditivo Central: Manual de Avaliação, ${ }^{6}$ volume 1 (faixa 4) e volume 2 (faixa 6), e o compact disc do RGDT desenvolvido por Keith, ${ }^{13}$ versão Auditec of Saint Louis (faixa número 2 à faixa número 8). A avaliação foi realizada em cabine acústica.

OSSW é composto por uma lista de 40 itens sendo que cada item é formado por quatro apresentações totalizando 160 palavras de quatro dissílabos cada. Os primeiros e os quartos dissílabos de cada item são apresentados isoladamente e separadamente a cada orelha, isto é, sem competição. Os segundos e terceiros dissílabos, porém, são apresentados dicoticamente, ou seja, simultaneamente às duas orelhas. Portanto, o teste possui quatro condições distintas: direito não competitivo (DNC), direito competitivo (DC), esquerdo competitivo (EC) e esquerdo não competitivo (ENC). Entre cada um dos itens, há um intervalo que permite a resposta verbal do paciente. $\mathrm{O}$ teste SSW foi realizado de acordo com o proposto por Borges ${ }^{14}$ e a intensidade utilizada na apresentação do estímulo foi de $50 \mathrm{dBNS}$, considerando-se a média tritonal aritmética dos resultados da audiometria tonal por via aérea nas frequências de $500 \mathrm{~Hz}, 1$ e $2 \mathrm{kHz}$. Os resultados foram analisados de maneira quantitativa e qualitativa.

Para a análise quantitativa, realizou-se o SSW simplificado (SSW-S) e analisou-se os erros para cada uma das condições das orelhas separadamente e computou-se a porcentagem de acertos. Considerou-se normal acertos iguais ou superiores a $90 \%$, nas condições de pesquisa DC e EC.?

Para a análise qualitativa, analisou-se as tendências de respostas encontradas, sendo elas: (a) efeito ordem (EO) - errar mais vezes nas duas primeiras palavras espondaicas ou nas duas últimas dos itens dos testes; (b) efeito auditivo (EA) - errar mais vezes quando o teste é iniciado pela orelha direita ou pela orelha esquerda; (c) tipo A - quando há um grande número de erros numa mesma coluna do SSW (coluna B ou F) e (d) inversões - quando as palavras de um item são repetidas fora de ordem..$^{14,15}$

O PSI é um teste de reconhecimento composto por 10 frases, para a identificação de figuras na presença de mensagem competitiva ipsilateral e contralateral. O PSI foi realizado utilizando a medida ipsilateral, com nível de apresentação do sinal da fala de $40 \mathrm{dBN} s .{ }^{16} \mathrm{O}$ paciente identifica uma figura correspondente à frase que foi apresentada na presença de mensagem competitiva ipsilateral nas relações fala/competição OdBNA e -10 dBNA. ${ }^{16} \mathrm{O}$ número de acertos foi convertido em porcentagem, considerando $10 \%$ para cada frase identificada corretamente. Considerou-se normal os acertos iguais ou superiores a 80\% na relação fala/competição 0 dBNA e 70\% na relação $-10 \mathrm{dBNA}$.

O RGDT consiste em pares de tons puros nas frequências de $500 \mathrm{~Hz}, 1,2$ e $4 \mathrm{kHz}$, com intervalos entre dois tons que variam de 0 a $40 \mathrm{~ms}$, podendo ser de $0,2,5$, $10,15,20,25,30$ ou $40 \mathrm{~ms}$, intervalos interestímulos de 4,5 segundos para permitir o tempo de resposta para o paciente. Os intervalos de sons são apresentados de forma aleatória. O procedimento é repetido em cada uma das frequências. Em todos os subtestes o indivíduo é orientado que ouvirá dois sons muito próximos e que deve levantar um dedo quando escutar um som e dois dedos quando escutar dois sons. $\mathrm{O}$ teste foi apresentado a $50 \mathrm{dBNS}$ nas frequências testadas na condição binaural. $\mathrm{O}$ resultado do RGDT foi medido por meio do menor intervalo a partir do qual o indivíduo passa a identificar a presença de dois estímulos. $\mathrm{O}$ resultado foi calculado para cada frequência, de 500 a $4 \mathrm{kHz}$, e também foi realizado o cálculo entre os resultados das quatro frequências. Considerou-se normal a média menor que $10 \mathrm{~ms}$.

Para a avaliação do potencial evocado auditivo de tronco encefálico foram utilizados dois canais, estímulo clique na intensidade de $90 \mathrm{dBnHL}$, polaridade alternada com frequência de apresentação de $21.1 \mathrm{c} / \mathrm{s}$, janela de $15 \mathrm{~ms}$, filtro de 30 a $3000 \mathrm{~Hz}$, e no mínimo 2000 estímulos e dois registros de reprodução. Foram utilizados eletrodos descartáveis da marca Kendall Medtrace $2000{ }^{\circledR}$ posicionados nas mastóides direita e esquerda, bem como um eletrodo posicionado em $\mathrm{Fz}$ (segundo o Sistema Internacional 10-20 de posicionamento de eletrodos), e o eletrodo terra na fronte. A impedância dos eletrodos foi mantida inferior a $5 \mathrm{k} \Omega$. Os cliques foram apresentados via fones de inserção $3 \mathrm{~A}$. No caso de presença das ondas I, III e V as mesmas foram identificadas e suas latências medidas, bem como, os intervalos interpicos I-III, III-V e I-V. O equipamento utilizado foi o Bio-logic's Evoked Potential System ${ }^{\circledR}$, em sala acusticamente e eletricamente tratada. O critério de análise seguido foi o proposto por Hall. ${ }^{17}$

O paciente foi submetido à avaliação vestibular através das seguintes provas: (a) pesquisa de nistagmo e de vertigem de posição/posicionamento através da manobra de Brandt e Daroff; ${ }^{18}$ (b) pesquisa de nistagmo espontâneo e semiespontâneo com os olhos abertos, no olhar frontal e a $30^{\circ}$ de desvio do olhar para a direita, esquerda, para cima e para baixo; (c) vectoeletronistagmografia (VENG) através de um aparelho termossensível, com três canais de registro, da marca Berger, modelo VN $316^{\circledR}$. Este tipo de VENG 
possibilitou obter medidas mais precisas da velocidade do componente lento (correção vestibular) do nistagmo. Utilizou-se uma cadeira rotatória pendular decrescente, um estimulador visual (Neurograff, modelo EV VEC ${ }^{\circledR}$ ), e um otocalorímetro a ar (Neurograff, modelo NGR 05 ${ }^{\circledR}$ ).

Segundo os critérios propostos por Padovan e Pansini ${ }^{19}$ e Mangabeira-Albernaz et al. ${ }^{20}$ foram realizadas as seguintes provas oculares e labirínticas à VENG: (a) calibração dos movimentos oculares; (b) pesquisa do nistagmo espontâneo (olhos abertos e fechados) e semiespontâneo (olhos abertos) - neste registro avaliou-se a ocorrência, direção, efeito inibidor da fixação ocular (EIFO) e o valor da velocidade angular do componente lento (VACL) máximo do nistagmo; (c) pesquisa do rastreio pendular para a avaliação da ocorrência e do tipo de curva; (d) pesquisa do nistagmo optocinético à velocidade de $60^{\circ}$ por segundo, nos sentidos anti-horário $(\mathrm{AH})$ e horário $(\mathrm{H})$, na direção horizontal, além da avaliação da ocorrência, direção, VACL máxima às movimentações anti-horária e horária do nistagmo; (e) pesquisa do nistagmo pré e pósrotatórios à prova rotatória pendular decrescente, com estimulação dos ductos semicirculares laterais, anteriores e posteriores (para a estimulação dos ductos semicirculares laterais [horizontais] a cabeça foi fletida $30^{\circ}$ para frente, e para a sensibilização dos ductos semicirculares anteriores e posteriores [verticais] o posicionamento da cabeça foi de $60^{\circ}$ para trás e $45^{\circ}$ à direita e, a seguir, $60^{\circ}$ para trás e $45^{\circ}$ à esquerda, respectivamente); (f) pesquisa do nistagmo pré e pós-calóricos realizada com o paciente posicionado de forma que a cabeça e o tronco estivessem inclinados $60^{\circ}$ para trás, para estimulação adequada dos ductos semicirculares laterais (o tempo de irrigação de cada orelha com ar a $42^{\circ} \mathrm{C}$ e $20^{\circ} \mathrm{C}$ durou 80 segundos para cada temperatura e as respostas foram registradas com os olhos fechados e com os olhos abertos para a observação do EIFO). Nesta avaliação verificou-se a direção, os valores absolutos da VACL e o cálculo das relações da preponderância direcional e predomínio labiríntico do nistagmo pós-calórico.

\section{RESULTADOS}

Avaliação audiológica: limiares auditivos dentro dos padrões de normalidade na orelha direita com perda neurossensorial a partir de $2 \mathrm{kHz}$ na orelha esquerda. Medidas de imitância acústica: curvas timpanométricas do tipo A com reflexo acústico ausente na medida ipsilateral em $2 \mathrm{kHz}$ à direita e em $4 \mathrm{kHz}$ bilateral e na medida contralateral ausente em $2 \mathrm{kHz}$ à esquerda e em $4 \mathrm{kHz}$ bilateral. Processamento auditivo central: interação binaural e localização sonora (3 acertos de 5 apresentações), análise e síntese (70\% de acertos), SSW (80\% de acertos na direita competitiva e 55\% de acertos na esquerda competitiva). Os resultados indicam dificuldade na habilidade de integração binaural. Observou-se padrão tipo A significativo em relação às tendências de respostas. PSI: apresentou 90\% de acertos na orelha esquerda e 100\% na orelha direita na relação fala/competição - 0 dBNA e $100 \%$ de acertos na orelha esquerda e $60 \%$ na orelha direita na relação fala/competição -10 dBNA. RGDT: apresentou média $(0,5 ; 1 ; 2$ e $4 \mathrm{kHz})$ de $4,75 \mathrm{~ms}$, demonstrando não haver dificuldade na habilidade de resolução temporal. Potencial evocado auditivo de troco encefálico: aumento de latência da onda I bilateralmente com consequente diminuição interpicos I-III e III-V. A onda V apresentou latência precoce bilateralmente. Avaliação vestibular: nistagmo de posição e/ou posicionamento ausente; calibração dos movimentos oculares regular; nistagmo espontâneo ausente com olhos abertos e com olhos fechados; nistagmo semiespontâneo ausente; rastreio pendular tipo II; nistagmo optocinético simétrico com VACL AH $=10 \%$ s; VACL $\mathrm{H}=11 \%$ \% ; nistagmo per-rotatório - estimulação dos ductos semicirculares laterais: simétricos com frequência nistágmica ( $\mathrm{AH}=9 ; \mathrm{H}=9 ; \mathrm{PDN}=0 \%$; estimulação dos ductos semicirculares posteriores: simétricos com frequência nistágmica $(\mathrm{AH}=8 ; \mathrm{H}=10 ; \mathrm{PDN}=11 \%$ à direita); estimulação dos ductos semicirculares anteriores: simétricos com frequência nistágmica $(\mathrm{AH}=9 ; \mathrm{H}=11 ; \mathrm{PDN}=10 \%$ à direita); nistagmo pós-calórico - prova realizada com ar $-\left(42^{\circ} \mathrm{C}\right.$ orelha direita $): \mathrm{VACL}=4 \% \mathrm{~s}\left(42^{\circ} \mathrm{C}\right.$ orelha esquerda): $\mathrm{VACL}=1 \stackrel{\circ}{\circ} \mathrm{s}\left(20^{\circ} \mathrm{C}\right.$ orelha direita $): \mathrm{VACL}=2 \% \mathrm{~s}$ ( $20^{\circ} \mathrm{C}$ orelha esquerda): $\mathrm{VACL}=7 \%$ s. Presença de EIFO nas quatro estimulações. $\mathrm{O}$ exame demonstra hiporreflexia em valor absoluto à prova calórica $42^{\circ} \mathrm{C}(\mathrm{OE})$, sugerindo síndrome vestibular periférica deficitária à esquerda.

\section{DISCUSSÃO}

Em relação aos achados audiológicos, observou-se perda auditiva do tipo neurossensorial de grau leve a moderado com curva descendente a partir de $2 \mathrm{kHz}$ na orelha esquerda, semelhante à presbiacusia. Sabe-se que o envelhecimento causa degeneração coclear que afeta, particularmente, a parte basal da cóclea, prejudicando a percepção auditiva nas altas frequências. ${ }^{21}$ Skinner e Miller $^{22}$ referem que as altas frequências são importantes para a discriminação consonantal e reconhecimento da fala.

A perda auditiva neurossensorial não pode ser considerada como o fator determinante das alterações do PA em idosos, porém elas podem representar um agravante. ${ }^{23}$ A timpanometria apresentou-se sem alterações de orelha média, sendo compatível com os limiares auditivos.

Segundo a American Speech Hearing Association (ASHA), considera-se um distúrbio do PAC quando se observa alteração em uma ou mais habilidades auditivas: localização e lateralização sonora, discriminação auditiva, 
reconhecimento de padrões auditivos, aspectos temporais da audição incluindo resolução, mascaramento integração e ordenação temporal, desempenho auditivo na presença de sinais competitivos e desempenho auditivo com sinais acústicos degradados. ${ }^{24} \mathrm{O}$ distúrbio do PA é uma disfunção específica dos processos auditivos, mas também pode estar associado à déficits de linguagem, memória e atenção.

No caso relatado, houve uma dificuldade na habilidade de localização sonora, no teste de interação binaural, designado para avaliar a habilidade do PAC em receber informações em ambas as orelhas e unificá-las em um evento perceptual. Esta dificuldade pode acontecer em patologias localizadas no tronco encefálico. ${ }^{25}$ As estruturas do SNC responsáveis por esta habilidade são o complexo olivar (recebe fibras das duas orelhas em cada lado e compara características sonoras entre ambas) e o córtex auditivo (utiliza diferenças de intensidade e tempo de chegada do som para decidir de onde vem o som). ${ }^{26}$

O traumatismo cranioencefálico e os acidentes vasculares cerebrais (hemorrágico ou isquêmico) são fatores etiológicos frequentemente associados aos distúrbios de linguagem, e que o teste SSW mostra-se útil na investigação topodiagnóstica dos pacientes portadores de lesão do córtex cerebral. ${ }^{27}$ Essas afirmações corroboram com os achados deste estudo, no qual a lesão no hemisfério esquerdo causado pelo AVC resultou na alteração do teste SSW sendo este pior na orelha esquerda com comprometimento da habilidade de integração devido à lesão no hemisfério ipsilateral.

No caso relatado por nós, a tendência de resposta na escuta dicótica foi padrão tipo A. A presença desta tendência demonstra a existência de comprometimento do corpo caloso, da região talâmica ou do giro angular. ${ }^{28}$

A presença de alteração no teste de escuta dicótica pode também estar relacionada com a perda auditiva periférica ou com a própria idade. Segundo Baran e Musiek ${ }^{29}$ os efeitos do envelhecimento podem envolver tanto a parte periférica quanto a central da audição e, quando o fator idade está relacionado diretamente com a perda auditiva periférica, esta pode estar dificultando ainda mais a escuta dicótica nos idosos..$^{30}$ Concordante com este estudo, Ortiz e Peroni ${ }^{31}$ no teste de escuta monótica de baixa redundância (PSI$\mathrm{MCI}$ ) na relação sinal/ruído em -10dBNA, encontraram maior erro na orelha direita, o que permite inferir que, a lesão em hemisfério esquerdo interferiu no processamento da informação auditiva, principalmente dos estímulos recebidos à direita. Os autores ${ }^{31}$ concluíram que os afásicos apresentaram dificuldades em desempenhar tarefas de figura-fundo, além de atenção seletiva para sons verbais em escuta de mensagem competitiva, tornando assim, relevante as avaliações que são capazes de fornecer dados sobre a compreensão dos mesmos nas situações comuns de comunicação que ocorrem diariamente.
Otsuki et al..$^{32}$ demonstraram que o PEATE é normal quando realizado em indivíduos afásicos. Discordante deste estudo, onde os registros evidenciaram aumento da latência da onda I bilateralmente, com consequente diminuição interpicos I-III e III-V, a onda V apresentou latência precoce bilateralmente. $\mathrm{O}$ aumento da latência absoluta da onda $\mathrm{V}$ e do interpico I-V é condizente com os relatos da literatura consultada, que menciona a possibilidade de aumento da latência da onda $\mathrm{V}$ em decorrência do fator idade. $8,33,34$

O equilíbrio corporal depende da integridade do sistema vestibular: labirinto, nervo vestibulococlear, núcleos, vias e inter-relações do SNC, do sistema somatossensorial e da visão. ${ }^{9} \mathrm{O}$ desequilíbrio surge quando algum fator interfere no funcionamento normal deste complexo sistema neurofisiológico.

O estudo do nistagmo realizado por meio da VENG temse constituído como um recurso preciso no topodiagnóstico de várias doenças. Nas síndromes otoneurológicas centrais, observa-se que os sinais de afecção do sistema vestibular central são predominantes em diversas provas labirínticas, fato que não ocorre nas síndromes periféricas. ${ }^{20}$

No caso relatado, ao exame vestibular, observouse alteração apenas na prova calórica, ocorrendo uma hiporreflexia labiríntica em valor absoluto à prova calórica $42^{\circ} \mathrm{C}$ à esquerda, com presença do efeito inibidor da fixação ocular. Não encontramos nenhum estudo para que pudéssemos confrontar com os nossos achados.

Nossos dados demonstram a importância da avaliação audiológica (periférica e central) e vestibular na contribuição dos procedimentos a serem realizados no acompanhamento terapêutico de pacientes com afasia de Broca.

\section{REFERÊNCIAS}

1. Coudry MI. Diário de narciso. São Paulo: Martins Fontes; 1986/ 1988.

2. Morato EM (org.). Afasia, interação e significação: as práticas discursivas de um centro de convivência de afásicos e não-afásicos. Campinas: Unicamp; 2003.

3. Bonini D. O papel da família na reabilitação do paciente afásico. Monografia de conclusão do curso de especialização em linguagem. CEFAC - Centro de Especialização em Fonoaudiologia Clínica. São Paulo; 1998.

4. Novaes-Pinto R, Santana AP. Semiologia nas afasias: implicações para a clínica fonoaudiológica. In: Mancopes R, Santana AP (orgs). Perspectivas na clínica das afasias: o sujeito e o discurso. Editora Santos; no prelo.

5. Madalozzo D. As correlações clínico-topográficas das afasias. Dissertação de Mestrado em Ciências da Saúde. Faculdade de Medicina de São José do Rio Preto. São José do Rio Preto, SP; 2007.

6. Pereira LD, Schochat E. Processamento auditivo central: manual de avaliação. São Paulo: Lovise; 1997.

7. Pereira LD. Processamento auditivo central: abordagem passo a passo. In: Pereira LD, Schochat E. Processamento auditivo central: manual de avaliação. São Paulo: Lovise; 1997. p. 49-59.

8. Alvarenga KF, Lamônica DC, Costa Filho AO, Banhara MR, Oliveira DT, Campo MA. Estudo eletrofisiológico do sistema auditivo periférico e central em um indivíduo afásico. Arq. Neuropsiquiatr 2005;63(1):104-9. 
9. Ganança MM, Caovilla HH, Munhoz MSL, Silva MLG, Frazza MM. As etapas da equilibriometria. In: Caovilla HH, Ganança MM, Munhoz MSL, Silva MLG. Equilibriometria clínica. 1. ed. São Paulo: Atheneu; 1999. p. 41-79.

10. Davis H, Silverman RS. Hearing and deafness. 3. ed. New York: Holt, Rinehart \& Wilson; 1970. p. 253-79.

11. Silman S, Silverman CA. Auditory diagnosis, principles and applications. London: Singular Publishing Group; 1991. p. 215-32.

12. Jerger J. Clinical experience with impedance audiometry. Arch Otolaringol 1970; 92:311-24.

13. Keith R. Random gap detection test. St. Louis, MO: Auditec; 2000.

14. Borges ACLC. Dissílabos alternados - SSW. In: Pereira LD, Schochat E. Processamento auditivo central: manual de avaliação. São Paulo: Lovise; 1997. p. 169-78.

15. Katz J, Ivey RG. Testes Centrais: Procedimentos utilizando espondeus. In: Katz J. Tratado de Audiologia Clínica. São Paulo: Manole; 1989. p. 237-53.

16. Zilioto KN, Kalil DM, Almeida CIR. PSI em português. In: Pereira LD, Schochat E. Processamento auditivo central: manual de avaliação. São Paulo: Lovise; 1997. p. 114-28.

17. Hall J. Handbook of auditory evoked responses. Boston: Allyn \& Bacon; 1992.

18. Brandt T, Daroff RB. Physical therapy for benign paroxysmal positional vertigo. Arch Otolaryngol 1980;106:484-5.

19. Padovan I, Pansini M. New possibilities of analysis in electronystagmography. Acta Otolaryngol 1972;73(2):121-5.

20. Mangabeira-Albernaz PL, Ganança MM, Pontes PAL. Modelo operacional do aparelho vestibular. In: Mangabeira-Albernaz PL, Ganança MM. Vertigem. 2.ed. São Paulo: Moderna; 1976. p. 29-36.

21. Macincuk MC. Inner ear, presbycusis. Texas: Department of Otolaryngology Head Neck Surgery, University of Texas Southwestern Medical Centre; 2002.

22. Skinner MV, Miller JD. Amplification handwidth and intelligibility of speech in quiet and noise for listeners with sensorioneural hearing loss. Audiology 1983;22:253-79.

23. Diveyi PL, Haupt KM. Audiological correlates of speech understanding deficits in elderly listeners with mild to moderate hearing loss. Ear Hear 1995;18:19-32.
24. ASHA - American Speech-Language-Hearing Association. Task force on central auditory processing consensus development: central auditory current status of research and implications for clinical practice. J Am Acad Audiol 1996;5:41-54.

25. Baran JA, Musiek FE. Avaliação comportamental do sistema nervoso auditivo central. In: Musiek FE, Rintelmann WF. Perspectivas atuais em avaliação auditiva. São Paulo: Manole; 2001.

26. Bonaldi LV, Angelis MAA, Smith RL. Hodologia do sistema auditivo. In: Pereira LD, Schochat E. Processamento auditivo central: manual de avaliação. São Paulo: Lovise; 1997. p. 19-25.

27. Teixeira RO. Diagnóstico topográfico neurológico: neuroimagem $x$ teste dicótico de dissílabos alternados - SSW. 2002. 59f. dissertação (mestrado em Distúrbios da Comunicação Humana) - Universidade Federal de Santa Maria, Santa Maria-RS; 2002.

28. Sanchez ML, Alvarez AMMA. Processamento auditivo central: avaliação. In: Costa SS, et al. Otorrinolaringologia: princípios e prática. 2. ed. Porto Alegre: Artmed; 2006. p. 191-202.

29. Baran JÁ, Musiek FE. Avaliação comportamental do sistema nervoso auditivo central. In: Musiek FE, Rintelmann WF. Perspectivas atuais em avaliação auditiva. Barueri: Manole; 2001. p. 371-409.

30. Marotta RMB, Quintero SM, Marone SAM. Avaliação do processamento auditivo por meio do teste de reconhecimento de dissílabos em tarefa dicótica SSW em indivíduos com audição normal e ausência de reflexo acústico contralateral. Rev Bras Otorrinolaringol 2002;68(2):254-61.

31. Ortiz KZ, Peroni C. Compreensão de fala em situação de mensagem competitiva em afásicos. Rev CEFAC. 2008;10(2):226-32.

32. Otsuki M, Soma Y, Sato M, Homma A, Tsuji S. Slowly progressive pure word deafness. Eur Neurol 1998;39:135-40.

33. Praamstra P, Stegeman DF, Kooijman S, Moleman J. Evoked potential measures of auditory cortical function and auditory comprehension in aphasia. J Neurol Sci 1993;115:32-46.

34. Hall J W. Auditory evoked response practices. In: Hall JW, (ED) Principles handbook of auditory evoked response. Needham Heights: Allyn \& Bacon; 1990. Part II. p. 263-305.

Endereço para correspondência:

Bianca Simone Zeigelboim

Programa de Pós-graduação em Distúrbios da Comunicação

Rua Sydnei Rangel Santos, 238 - Santo Inácio

CEP 82010-330, Curitiba, PR, Brasil 